\title{
Intervenção precoce e autismo: um relato sobre o Programa Son-Rise
}

Early intervention end autism: a report about the Son-Rise Program

La intervención temprana y autismo: un informe sobre el Programa Son-Rise

\author{
Carlo Schmidt \\ Cristiane Kubaski* \\ Joise de Brum Bertazzo ${ }^{* * *}$ \\ Livia de Oliveira Ferreira ${ }^{* * *}$
}

\begin{abstract}
Resumo
A necessidade de intervenção precoce para pessoas com diagnóstico de autismo tem sido reiteradamente confirmada pela literatura. No entanto poucas experiências sobre programas de intervenção têm sido publicadas na literatura nacional, o que dificulta identificar o modo como são desenvolvidas bem como o impacto dessas práticas no desenvolvimento da criança. Este estudo tem por objetivos descrever a condução do Programa Son-Rise com uma criança com autismo durante 12 meses e o impacto dessa intervenção sobre o desenvolvimento da criança. Os resultados mostraram que o desenvolvimento do programa na residência da família ocasionou interferências na rotina familiar, diminuindo a intensidade da intervenção. Além disso, foram observados avanços mais significativos nas áreas da comunicação e interação do que no uso do olhar e contato visual. Por fim são discutidos os pontos positivos e limitações observados nesse processo.
\end{abstract}

Palavras-chave: Autismo. Intervenção precoce. Desenvolvimento.

\begin{abstract}
Early intervention for children and youth with autism has been confirmed by the literature. However, few experiences on intervention programs have been published in national literature, making it difficult to identify how they are developed and the impact of these practices on child development. This study aims to presents the running of Son-Rise Program with a child
\end{abstract}

\footnotetext{
Texto recebido em maio 2013 e aprovado para publicação em setembro de 2015.

* Professor do Departamento de Educação Especial da Universidade Federal de Santa Maria (UFSM). Endereço: Rua João Goularte, 501/302 - Bairro Camobi, Santa Maria-RS. CEP: 97105-220. E-mail: carlopsico2@gmail.com.

* Mestra em Educação pelo Programa de Pós-Graduação em Educação da UFSM. Endereço: Rua Ricardo Schmidt, 38 - Nossa Senhora de Lourdes, Santa Maria-RS. CEP: 97050-235. E-mail: kubaski229@bol.com.br.

*** Mestra em Educação Especial pelo Programa de Pós-Graduação em Educação da UFSM. Endereço: Rua Venâncio Aires, 576, ap. 303 - Passo d'Areia, Santa Maria-RS. CEP: 97010-000. E-mail: joisebertazzo@gmail.com

${ }^{* * * *}$ Graduada em Educação Especial na UFSM, professora de Educação Especial e pesquisadora na FCEE. Endereço: Rua Antônio Scherer, 330, ap. 106 - Kobrasol, São José-SC. CEP: 88102-090. E-mail: livia_o@hotmail.com.
} 
with autism over twelve months, and the impact of this intervention on child development. Results showed that the program caused interferences in family routine, which caused decrease of the intensity of the intervention. Furthermore, significant advances were observed in Communication and Interaction areas more than in Use of Sight and Eye Contact. Finally we discuss the strengths and limitations noted in this process.

Keywords: Autism. Early intervention. Development.

\section{Resumen}

En este trabajo proponemos un instrumento para la escritura de caso clínico de niños pensado a partir de discusiones en el Núcleo de Enseñanza, Investigación y Extensión en Clínica Interdisciplinar de la Infancia de la Clínica de Tratamiento Psicológico de la Universidad Federal de Rio Grande do Sul. Nos apoyamos en trabajos sobre la importancia del registro para la práctica psicoanalítica y para la formación de nuevos terapeutas. Destacamos la particular relevancia de la escritura de caso para la clínica-escuela, donde la temática de la formación es fundamental. De los debates en el Núcleo y apoyados en la teoría de la constitución del sujeto, derivamos tres ejes capaces de orientar la escritura de los casos y de respetar singularidades: el niño en el discurso parental, aquello que él capturó para sí mismo de lo que le fue ofrecido, su inserción en el lazo social. La utilización de la ficha de registro, después de superar resistencias iniciales, se mostró un potente y adecuado instrumento para las finalidades propuestas.

Palabras clave: Transmisión. Relato de caso. Psicoanálisis con niños. Clínica escuela.

\section{Introdução}

autismo pode ser definido como um complexo transtorno neurocomportamental que inclui prejuízos na interação social, comunicação, linguagem e comportamentos estereotipados (Eeghen et al., 2013). Conforme o DSM-IV, o autismo pertence à categoria dos transtornos globais do desenvolvimento (TGD), juntamente com o transtorno de Rett, transtorno desintegrativo da infância, transtorno de Asperger e transtorno invasivo do desenvolvimento sem outra especificação - TID-SOE (Associação Psiquiátrica Americana [APA], 2002).

O fenótipo comportamental das pessoas com TGD se manifesta de formas extremamente heterogêneas, resultando na tendência atual em integrar todas essas categorias sob a terminologia de transtornos do espectro do autismo (TEA) (APA, 2013). Desse modo, as pessoas com TEA apresentam alteraçôes importantes nas áreas da comunicação e interação sociais, comportamental, 
além de diversos outros aspectos, como alterações sensoriais (ex.: hipo ou hipersensibilidade auditiva, tátil, visual), comorbidades (ex.: deficiência mental, síndrome do x-frágil) e peculiaridades que interferem diretamente sobre o modo como aprendem (ex.: dificuldades de imaginação, generalização do aprendizado e inflexibilidade cognitiva) (Schmidt, 2012).

Essas peculiaridades levaram pesquisadores e investigar e a propor alternativas que oferecessem respostas mais efetivas para o ensino e aprendizagem dessas crianças, incluindo desde adaptações curriculares até estratégias de ensino, constituindo programas de intervenção (Tutt, Powell \& Thornton, 2006). Atualmente há evidências claras de que esses programas podem promover avanços importantes nas áreas da linguagem, cognição, atividades de vida diária, contribuindo para uma melhor qualidade de vida desses indivíduos e de suas famílias (Remington et al., 2007).

Cada programa apresenta orientação e práticas diferenciadas entre si, podendo vir a constituírem-se como abordagens educacionais, terapêuticas, filosofias ou serviços de atenção. Por exemplo, os programas de orientação psicoeducacional têm como foco comum promover o desenvolvimento daqueles comportamentos que as crianças aprendem "naturalmente", mas aquelas com autismo necessitam de um ensino especial. Podem ser destacadas as Escolas Higashi (Sandberg \& Spritz, 2012), escolas orientadas pelo método montessoriano (Flowers, 1993), o LEAP (learning experiences) (Strain \& Bovey, 2011) e o TEACCH (treatment and education of autistic and related communication handicapped children) (Welterling, Turner-Brown, Harris, Mesibov \& Delmolino, 2011).

As abordagens de base exclusivamente comportamental têm sido as mais frequentemente descritas na literatura no ensino de pessoas com TEA, como é o caso do ABA (Applied Behavior Analysis) e suas derivações: treinamento de ensaios discretos (Holding, Bray \& Kehle, 2011), treinamento de comunicação funcional (Franco, Lang, O’Reilly, Chan, Sigafoos \& Rispoli, 2009) e treinamento de teoria da mente (Hollander \& Anagnostou, 2007).

Por fim, outros programas, chamados desenvolvimentistas, direcionam o foco da intervenção diretamente para o desenvolvimento da criança, tal como o Floor Time (Greenspan \& Wieder, 2000), o SCERT (Social Communication, Emotional Regulation, Transactional Support) (Prizant, Wetherby \& Rydell, 2000) e o Son-Rise (Kaufman, 1976). Estes têm por objetivo favorecer o desenvolvimento da linguagem e habilidades de comunicação funcional em ambientes naturais, por meio da retomada da sequência do desenvolvimento típico inicial, de modo a maximizar as condutas intencionais e socioafetivas da criança. 
Nota-se, portanto, a existência de uma variedade de possibilidades de intervenção para crianças com autismo. Entre estas, o Programa Son-Rise tem recebido bastante atenção recentemente, tanto da mídia quanto na literatura científica brasileira (Botti \& Cota, 2011; Suplino, 2010).

\section{O programa Son-Rise}

Também conhecido como Option Method, no Reino Unido, o Programa SonRise (SRP) foi originalmente desenvolvido nos Estados Unidos, na década de 1970 (Kaufman, 1976, 1994). O SRP é geralmente implantado e conduzido pelos pais, auxiliados por facilitadores ${ }^{1}$ os quais recebem formação do Autism Treatment Center of America (ATCA) ${ }^{2}$ ou por alguém credenciado por esse local.

O programa é desenvolvido na casa dos pais, em um quarto modificado para ser o "quarto de brincar". Esse ambiente é adaptando estrategicamente, a fim de destacar a organização e a previsibilidade, minimizar distrações e obter maior controle sobre possíveis mudanças, visando a maximizar a interação com a criança com autismo (Deisinger Burkhardt, Wahlberg, Rotatori \& Obiakor, 2012).

As estratégias educacionais do SRP orientam para que o adulto, por meio de uma interação $1: 1$, siga os interesses da criança, em vez de direcioná-los a uma atividade proposta, valorizando os comportamentos iniciados pelo filho, mesmo aqueles socialmente inadequados, como as estereotipias. O objetivo seria a busca de atitudes que reflitam valores de aceitação e ausência de julgamento (Kaufman, 1994).

Nos momentos em que a criança apresenta maior responsividade, cabe ao adulto propor a expansão das atividades conjuntas com vistas a promover $o$ desenvolvimento das habilidades de comunicação e interação social (Williams, 2006).

Quanto à intensidade e frequência da intervenção, o ATCA recomenda acima de 20 horas semanais de intervenção, ao passo que os workshops ministrados no Brasil sugerem iniciar o SRP com 30 minutos diários, aumentando gradativamente (Tolenzani, 2011; Williams \& Wishart, 2003). Algumas famílias utilizam o programa de modo ainda mais intensivo, acima de 80 horas semanais em alguns casos, solicitando para isso a participação de voluntários (Kaufman, 1994). No entanto, conforme o estudo de Williams e Wishart (2003), a maioria

1 Facilitador é o nome utilizado pelo SRP para designar a pessoa que está trabalhando com a criança, podendo ser os pais ou outros adultos recrutados para auxiliar a família.

2 O Centro de Tratamento para Autismo da América (ATCA) localiza-se em Massachussets, EUA, e constitui-se como a sede do Programa Son-Rise. 
dos pais que se propõem a realizar o programa Son-Rise com seu filho planeja utilizar mais tempo na intervenção do que realmente conseguem quando esta se inicia.

Percebe-se que há uma escassez de pesquisas investigando a forma como são conduzidos esses programas de intervenção e seus resultados, especialmente no Brasil, o que dificulta identificar o impacto dessas práticas. Justamente devido à ausência dessas pesquisas, o SRP é considerado uma intervenção com informações de validade limitada, ou seja, não há evidências que apoiem sua indicação (Odom, Boyd, Hall \& Hume, 2010; Simpson, 2005).

A obtenção de informações sobre diferentes intervenções em autismo possibilita que os pais optem por programas que considerem as especificidades do seu filho, evitando assim investimento financeiro e de tempo com uma intervenção inadequada.

Este estudo apresenta o acompanhamento de uma intervenção com o Programa Son-Rise, o qual foi conduzido pelos pais de uma criança com autismo e por facilitadores, na residência daqueles, ao longo de um ano. São apresentados dados sobre a implantação e a condução desse programa, bem como o impacto no desenvolvimento da criança, para discutir os pontos positivos e limitações observadas nesse processo.

\section{Metodologia}

\section{Participantes}

Participaram deste estudo uma família composta por pai, mãe, um filho com diagnóstico de autismo e a irmã. O recrutamento foi realizado por conveniência, tendo como critérios de inclusão a configuração de família nuclear composta por pai, mãe e um ou mais filhos, sendo que um deles deveria ter o diagnóstico de transtorno autista (APA, 2002). Os pais realizaram a capacitação para intervenção com o Programa Son-Rise - Nível I $(\text { start-up })^{3}$ cerca de um ano antes do início da implantação do programa em sua residência.

O pai, 35 anos, é militar, enquanto a mãe, 30 anos, é técnica de enfermagem. O filho com autismo tem quatro anos e frequenta uma escola municipal no turno da tarde. Por essa razão, a intervenção foi realizada no turno da manhã e em algumas noites, concomitantes à sua frequência na escola. Sua irmã tem 11 anos e frequenta a $5^{\circ}$ ano do ensino fundamental.

3 O treinamento nível 1 é um curso de 15 horas ministrado por uma pessoa credenciada ao SRP, que habilita a família a recrutar os facilitadores e iniciar a intervenção em sua residência. 
O protocolo dessa pesquisa foi aprovado pelo Comitê de Ética em Pesquisa UFSM, sob o processo número 23081.003012/2010-19.

\section{Procedimentos}

O casal participante organizou o quarto de brincar conforme as especificações do SRP, em um dos cômodos de sua residência, onde transcorreu toda a intervenção. As facilitadoras visitavam a família para realizar a intervenção cinco manhãs por semana, com duração entre uma e duas horas, sendo uma facilitadora para cada dia.

A intervenção Son-Rise utiliza três instrumentos desenvolvidos pela ATCA para avaliar o desenvolvimento da criança ao longo da intervenção:
a) modelo de desenvolvimento;
b) currículo social; e
c) relatório semanal. ${ }^{4}$

Antes do início da intervenção, as facilitadoras e os pais realizaram a avaliação da criança utilizando o modelo de desenvolvimento do SRP. Trata-se de um instrumento para identificação do estágio de desenvolvimento das habilidades em cada dimensão (Hogan \& Hogan, 2007).

O modelo apresenta quatro dimensóes do desenvolvimento:
a) contato visual;
b) comunicação;
c) atenção compartilhada; e
d) flexibilidade.

Para cada dimensão, há uma progressão do desenvolvimento das habilidades em cinco estágios: 
a) (1) habilidades básicas;

b) 2 e 3) habilidades intermediárias;

c) 4 e 5) habilidades avançadas). O estágio inicial em cada dimensão foi definido com base na média das observações dos pais e facilitadoras sobre as habilidades da criança.

A partir desta avaliação, foi elaborado o currículo social do SRP, o qual se constitui em uma ou duas metas para cada uma das quatro dimensóes, de acordo com o nível de habilidades da criança. Estas foram reavaliadas mensalmente mediante pontuações de aquisição total (adquirida), aquisição parcial (em desenvolvimento) ou não aquisição.

Em cada sessão com a criança, os facilitadores e pais centravam-se nas metas estabelecidas em cada dimensão, realizando um registro contendo dados sobre as atividades desenvolvidas com a criança e estratégias mais efetivas para atingi-las. Esses registros foram compilados semanalmente, gerando o relatório semanal do SRP, no qual constavam de metas e estratégias que se mostraram mais efetivas para atingi-las.

Quando uma meta era atingida com a criança, estipulava-se como nova meta a habilidade imediatamente subsequente, segundo o modelo de desenvolvimento do SRP. Desse modo, foi registrada a progressão da criança ao longo dos estágios de habilidades.

Além dos instrumentos próprios do programa Son-Rise, utilizou-se da escala CARS antes do início da intervenção e 12 meses depois. A childhood autism rating scale (CARS) é um instrumento amplamente utilizado na pontuação da intensidade dos sintomas no autismo, incluindo domínios como relacionamento interpessoal, comunicação, modalidades sensoriais e reações emocionais. Validada para uso com a população brasileira (Pereira, Riesgo \& Wagner, 2008), a escala é composta por 15 itens que são pontuados de 1 (limite da normalidade) a 4 (sintomas graves). A soma da pontuação de todos os itens permite a categorização seguinte: $<30=$ normal; $>30>36,5=$ autismo leve a moderado; $>37=$ autismo severo (Schopler, Reichler \& Renner, 1988).

Câmeras de vídeo foram instaladas no interior do quarto, permitindo a observação e posterior discussão entre avaliadores na busca de consenso quanto às pontuações individuais. Para fins dessa análise, os dados dos instrumentos do SRP foram agrupados por trimestre de intervenção, gerando quatro avaliações do desenvolvimento ao longo de 12 meses. 


\section{Resultados}

Os resultados são apresentados em duas partes. A primeira descreve o processo de implantação do programa pela família, incluindo dados como a intensidade e a frequência de intervenção pelos participantes. A segunda parte mostra dados do impacto da intervenção sobre o desenvolvimento da criança.

\section{Implementação e condução do programa}

Quanto à intensidade da intervenção, foram despendidas 256 horas e 33 minutos com a criança no quarto de brincar ao longo dos 12 meses. A média de horas diárias variou com a mínima de 1 hora e 42 minutos e a máxima de 2 horas e 10 minutos, obtendo a média geral de 1 hora e 54 minutos por dia, conforme a tabela 1 .

Tabela 1. Dados sobre a intensidade da intervenção

\begin{tabular}{cc}
\hline Intensidade da intervenção & Horas \\
\hline Total (12 meses) & $256 \mathrm{~h} 33 \mathrm{~min}$ \\
Média semanal & $6 \mathrm{~h} 37 \mathrm{~min}$ \\
Média diária & $1 \mathrm{~h} 54 \mathrm{~min}$ \\
\hline
\end{tabular}

Fonte: elaborado pelos autores.

A família participante pretendia, antes de iniciar a intervenção, utilizar o quarto com a criança durante duas a três horas diárias, ao passo que a média final de horas ao dia não chegou a duas. As razões justificadas pela família dizem respeito à interferência do programa na vida familiar, pelo qual a criança demorou algum tempo para ajustar-se aos novos horários, ao quarto de brincar e à presença constante das facilitadoras.

Participaram, ao todo, oito facilitadoras, além dos pais da criança com autismo. Entre estes, observa-se que o pai foi o mais participativo, seguido pelas facilitadoras 1 e 2, respectivamente. Dados sobre os demais participantes podem ser observados na tabela 2 . 
Tabela 2. Frequência das intervenções quanto aos participantes

\begin{tabular}{lc}
\hline \multicolumn{1}{c}{ Participantes da intervenção } & $f$ \\
\hline Pai & 35 \\
Facilitadora 1 & 30 \\
Facilitadora 2 & 21 \\
Facilitadora 3 & 14 \\
Facilitadora 4 & 12 \\
Mãe & 9 \\
Facilitadora 6 & 7 \\
Facilitadora 7 & 7 \\
Facilitadora 8 & 3 \\
Facilitadora 5 & 3 \\
\hline TOTAL & 141 \\
\hline
\end{tabular}

Fonte: elaborado pelos autores.

A maior participação do pai nas intervenções era esperada, já que este foi o principal idealizador do programa entre o casal e protagonista na organização e condução da intervenção. Por outro lado, observou-se a baixa frequência de intervençôes da mãe, a qual participou quase quatro vezes menos que o pai.

\section{Impacto da intervenção sobre o desenvolvimento da criança}

O resultado da CARS mostrou que a pontuação total era de 36,7 pontos antes da intervenção, correspondendo ao diagnóstico de autismo moderado. Observa-se que as áreas mais severamente afetadas foram comunicação verbal $(3,92)$, uso do objeto $(2,67)$ e adaptação a mudanças $(2,58)$, ao passo que as mais brandas foram uso do olhar $(2,17)$ comunicação não verbal (2) e paladar, olfato e tato $(2,08)$.

Ao comparar as pontuações pré e pós-intervenção, observa-se que a criança obteve redução na severidade dos sintomas, conforme dados da tabela 3 .

Tabela 3. Médias das pontuações da CARS* antes e depois da intervenção

\begin{tabular}{l|l|l}
\hline Itens & CARS 1 & CARS 2 \\
\hline Relacionamento & 2,42 & 2,1 \\
\hline Imitação & 2,33 & 1,7 \\
\hline Resposta emocional & 2,42 & 2,1 \\
\hline Expressão corporal & 1,58 & 2,1 \\
\hline
\end{tabular}




\begin{tabular}{l|l|l}
\hline Uso do objeto & 2,67 & 2,2 \\
\hline Adaptação a mudanças & 2,58 & 2,5 \\
\hline Uso do olhar & 2,17 & 2,3 \\
\hline Uso da audição & 2,42 & 1,2 \\
\hline Paladar, olfato e tato & 2,08 & 1,4 \\
\hline Medo e nervosismo & 2,42 & 1,7 \\
\hline Comunicação verbal & 3,92 & 3,2 \\
\hline Comunicação não verbal & 2,00 & 2,1 \\
\hline Atividade & 2,42 & 2,5 \\
\hline Inteligência & 2,25 & 2 \\
\hline Impressão geral & 2,83 & 2,4 \\
\hline Total** & 36,7 & 31,5 \\
\hline
\end{tabular}

* 1 a 1,5: normal; 2 a 2,5: leve; 3 a 4: moderado.

** < 30 pts: normal; > $30<36,5$ pts: autismo leve a moderado; > 37 pontos: autismo severo

Fonte: elaborado pelos autores.

No escore geral da CARS, a criança apresentou uma pontuação pré-intervenção equivalente ao diagnóstico de autismo moderado a severo (36,7 pontos), o qual obteve redução ao final da intervenção para autismo leve (31,5 pontos.).

Essa diminuição da intensidade geral dos sintomas contemplou 11 das 15 áreas avaliadas, em que a área sensorial (audição, paladar, tato e olfato), medo/ nervosismo e comunicação verbal obtiveram as melhorias mais sensíveis. Porém quatro áreas não apresentaram diminuição nos sintomas durante a intervenção: expressão corporal, uso do olhar, comunicação não verbal e atividade.

Os dados do modelo de desenvolvimento SRP foram agrupados em quatro avaliações trimestrais, permitindo a análise da progressão nos estágios e metas. Quanto aos estágios, o resultado da avaliação pré-intervenção mostra que todos os comportamentos pertencentes ao estágio 1 estavam presentes nas áreas de atenção compartilhada e flexibilidade, fazendo com que se iniciasse a intervenção a partir do estágio dois. Já os comportamentos de comunicação verbal e Contato visual encontravam-se parcialmente completos, iniciando no estágio 1.

Quanto às metas, observa-se que estas foram atingidas em maior frequência nas habilidades de atenção compartilhada, totalizando 10 ao longo da intervenção, seguida pelas habilidades de contato visual que completou 6 metas. As habilidades que menos progrediram foram as de comunicação verbal, com 3 metas atingidas, e flexibilidade, com apenas 1. Esses dados podem ser observados na figura 1. 


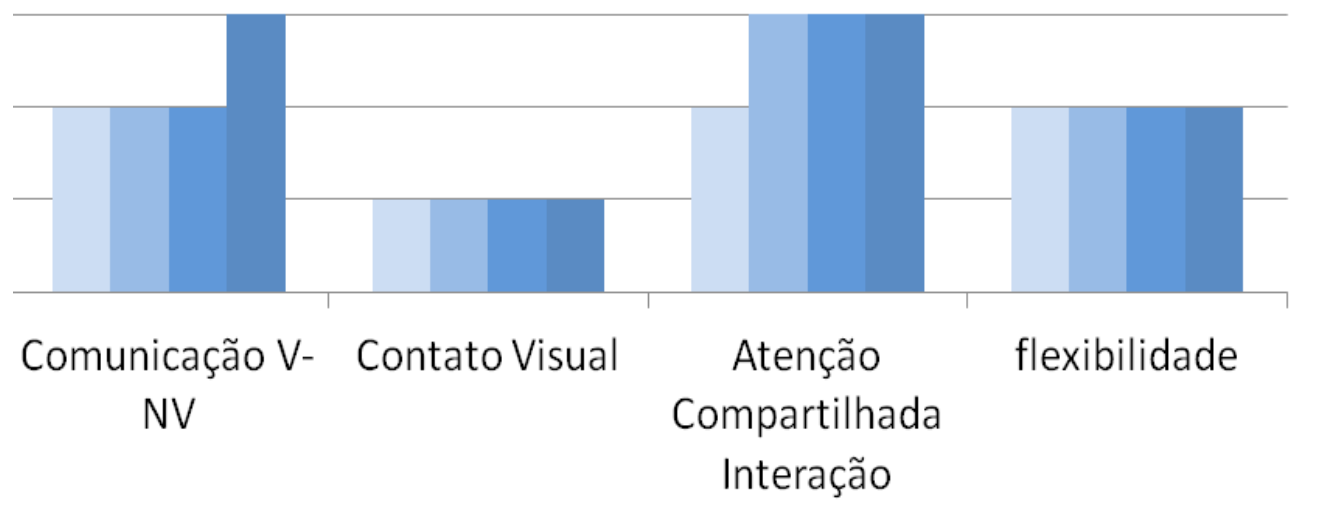

Fonte: elaborado pelos autores.

Ao comparar os resultados das avaliações da CARS com o dos relatórios SRP, observa-se que ambas as medidas identificaram tanto avanços quanto estagnações importantes no desenvolvimento da criança. Enquanto a CARS mostra a diminuição de 0,7 pontos na intensidade dos sintomas na área da comunicação verbal e um aumento de 0,1 na severidade na comunicação não verbal, os relatórios do Son-Rise também apontam avanços adquiridos na comunicação de modo geral, tanto verbal como não verbal, integrando essas dimensões.

Do mesmo modo, entende-se que a diminuição na intensidade dos sintomas na área de relacionamento, pela CARS, pode ser vista como equivalente aos avanços identificados pelos relatórios SRP no fundamento da atenção compartilhada. Isso porque muitos dos comportamentos que regulam a interação nas relações sociais são comportamentos de atenção compartilhada (ex.: direcionar o olhar, compartilhar interesse por objetos ou eventos), portanto corroborando os avanços obtidos.

Por outro lado, foram observados pouco ou nenhum avanço em outras áreas. Por exemplo, parece haver uma relação entre a não diminuição dos sintomas no item uso do olhar pela CARS e a falta de avanços no fundamento contato visual dos relatórios SRP. A CARS mostra que a severidade dos sintomas antes da intervenção manteve-se praticamente a mesma após um ano de programa, corroborando os relatórios SRP, que revelam que a criança não evoluiu além do nível 1 nesse fundamento. 


\section{Discussão final}

Este estudo descreveu a implantação e condução de um programa de intervenção sobre o autismo para, com isso, oferecer aos pais e profissionais um relato dessa experiência. Neste caso, foi descrita a forma como uma família aplicou o programa SRP com o filho, permitindo apresentar algumas conclusões.

O programa Son-Rise, sumariamente, apoia-se na filosofia de que mais do que obrigar a pessoa com autismo a participar das nossas vivências, os pais devem participar do mundo da criança como uma forma de construir uma ponte entre a criança e seus cuidadores. Uma vez que essa possibilidade é construída, o filho pode ser encorajado a avançar e experimentar experiências mais significativas e complexas.

Os criadores do SRP acreditam que esses princípios foram responsáveis por transformar seu filho não verbal, isolado e com um rebaixamento intelectual significativo em uma pessoa de fala fluente, social e extremamente inteligente. De fato, Raun Kaufman progrediu da escola para a universidade e tornou-se diretor de um centro de educação para crianças, o que justifica seu sucesso. Porém parece haver dúvidas sobre a fidedignidade desse diagnóstico, já que as bases neurobiológicas do autismo estão bem documentadas na literatura (Siegel, 1996; Pinto, Pagnamenta, Klei, Anney, Merico \& Regan, 2010). Independentemente de o diagnóstico estar ou não correto, parece difícil atribuir todo o sucesso posterior unicamente a essa intervenção.

Além disso, embora o ATCA não garanta que seu programa reabilite completamente ou cure pessoas com autismo, a reversão é claramente sugerida de diversas formas, por meio dos títulos dos livros (ex.: A miracle to believe in, Miracle continues), nas camisetas que as crianças sob esse tratamento usam, exibindo a frase "Miracle in progress" e nos portôes de entrada do Option Institute, onde se lê "A place for miracles" (Howlin, 1997).

Por outro lado, esse programa também conta com milhares de depoimentos de crianças e adultos que vêm utilizando o Son-Rise e descrevendo ganhos além das expectativas convencionais, algumas delas relatando melhorias significativas. Além do caso dos Kaufman, uma diversidade de relatos oriundos de várias fontes referencia a intervenção como positiva e efetiva no caso de pessoas com autismo.

Sobre esse aspecto, Schreibman (2007) sugere cautela na análise desses relatos, alertando para o fato de que esses resultados não podem ser confundidos com evidências de eficácia de tratamento, mas opiniōes de usuários do programa. 
Quanto ao impacto desse programa no desenvolvimento da criança, as avaliações continuadas apontaram avanços em determinadas áreas do desenvolvimento (ex.: comunicação, interação) e pouco ou nenhum avanço em outras (ex.: uso do olhar, contato visual), identificadas tanto pelos relatórios próprios do SRP quanto pela CARS. Nesse sentido, é importante destacar que, embora tenha se verificado uma progressão no quadro, não é impossível atribuir tais avanços apenas ao uso do programa, pois se desconsideraria o papel da escola, família e maturação no desenvolvimento infantil sobre o desenvolvimento da criança. Além disso, a quantidade de tempo de intervenção direta com a criança ao longo dos 12 meses encontrou-se abaixo do que é indicado pelo programa, o que teoricamente geraria ganhos pouco significativos.

Quanto ao arcabouço teórico que fundamenta a intervenção, percebese que é bastante extensa e derivada de sólidas evidências empíricas sobre desenvolvimento infantil e autismo (Houghton, 1997). Contudo é prudente destacarmos que essas pesquisas não dizem respeito à utilização do SRP, mas sobre seus fundamentos estudados isoladamente (ex.: atenção compartilhada, orientação social no autismo). Ainda não existem pesquisas sobre esse método que possam corroborar sua eficácia no tratamento de pessoas com autismo.

Já sobre o papel do adulto nas interações com a criança com autismo no quarto de brincar, Jordan e Powell (1993) afirmam que, apesar de esse programa postular que nenhum comportamento da criança deve ser julgado (mas aceito), na prática, alguns deles são deliberadamente ignorados, enquanto outros são recebidos entusiasticamente. Por essa razão, Jordan e Powell (1993) a caracterizada como uma prática intervencionista, já que são utilizadas recompensas para reforçar comportamentos desejados e extinção para extinguir os indesejáveis.

Por exemplo, quando em interação no quarto de brincar, é indicado que o adulto não reaja se a criança bater nele, por outro lado, o adulto deve reagir entusiasticamente diante das iniciativas de interação da criança. Apesar de não se tratar de um reforço primário com objetos tangíveis (ex.: alimentos), o condicionamento comportamental no SRP opera por meio de reforço secundário, por meio da satisfação de necessidades secundárias ou sociais (ex.: elogios, afagos) (Rosenwasser \& Axelrod, 2002). Com isso, o modelo do SRP parece teoricamente mais próximo de uma abordagem comportamental do que desenvolvimentista.

Por fim, atenção especial deve ser dada à forma como as famílias dedicarão seu tempo a esse programa. A família participante decidiu utilizar uma média de cinco facilitadoras por semana e avaliou essa escolha de modo ambíguo. Por um lado, a variedade de pessoas contribuiu para flexibilizar alguns comportamentos 
do filho e auxiliar no relacionamento; por outro, causou estresse nas relações conjugais. $\mathrm{O}$ casal relatou uma restrição na intimidade do casal devido à presença constante de pessoas externas ao ambiente familiar, além da falta de tempo para dedicar-se à outra filha, irmã do menino com autismo.

De fato, há evidências de que a implementação do SRP pode acarretar efeitos negativos sobre a família. Williams e Wishart (2003) concluíram que um grupo de pais que utilizou o Son-Rise durante 20 horas semanais relatou intenso estresse por sentirem-se cansados e por terem pouco tempo para convivência com os outros membros da família. Outro estudo corrobora esse argumento e acrescenta que intervenções como o SRP podem ser altamente invasivas devido à relação de proximidade estreita e contínua da criança (Schoen, 2003).

Enfim, os benefícios e críticas apresentados aqui sobre essa intervenção reforçam a necessidade do desenvolvimento de estudos mais rigorosos, de modo a endereçá-la mais precisamente, indicando claramente os principais riscos e benefícios.

\section{Referências}

Associação Psiquiátrica Americana. (2002). Manual diagnóstico e estatístico de transtornos mentais. Porto Alegre: Artes Médicas.

Associação Psiquiátrica Americana. (2013). Manual diagnóstico e estatístico de transtornos mentais. Porto Alegre: Artes Médicas.

Botti, N. \& Cota, F. (2011). Cinema e psiquiatria: filmes para o estudo do autismo. Revista de Enfermagem do Centro Oeste Mineiro, 1 (3), 313-323.

Deisinger, J., Burkhardt, S., Wahlberg, T., Rotatori, A. \& Obiakor, F. (2012). Autism spectrum disorders: inclusive communityfor the twenty-first century. Charlotte: Information Age Publishing.

Eeghen, A., Pulsifer, M., Merker, V., Neumeyer, A., Eeghen, E., Thibert, R., . . . \& Thiele, E. (2013). Understanding relationships between autism, intelligence, and epilepsy: a cross-disorder approach. Developmental Medicine \& Child Neurology, 55, 146-153.

Flowers, T. (1993). autism and montessori: old wisdom, new ideas. NAMTA Journal, 18 (1), 141-150. 
Franco, J. H., Lang, R. L., O’Reilly, M. F., Chan, J. M., Sigafoos, J. \& Rispoli, M. (2009). Functional analysis and treatment of inappropriate vocalizations using a speech-generating device for a child with autism. Focus on Autism and Other Developmental Disabilities, 24 (3), 146-155.

Greenspan, S. \& Wieder. (2000). A developmental approach to difficulties in relating and communicating in autism spectrum disorders and related syndromes. In M. Wetherby \& B. Prizant (Eds.). Autism spectrum disorders: a transactional developmental perspective. (pp. 279-306). Baltimore: Paul H. Brookes.

Hogan, W. \& Hogan, B. (2007). Developmental model \& social curriculum. Massachussets, USA: The Option Institute \& Fellowship.

Holding, E., Bray, M. \& Kehle, T. (2011). Does speed matter? A comparison of the effectiveness of fluency and discrete trial training for teaching noun labels to children with autism. Psychology in the Schools, 48 (2), 166-183.

Hollander, E. \& Anagnostou, E. (2007). Clinical manual for the treatment of autism. Arlington: American Psychiatric Public.

Houghton, K. (1997). Pesquisas empíricas de apoio ao programa son-rise. Florianópolis: Inspirados pelo Autismo. Recuperado a partir de http://www. luzimarteixeira.com.br/wp-content/uploads/2009/11/autismo-metodo-sonrise.pdf

Howlin, P. (1997). Prognosis in autism: do specialist treatments affesct longterm outcome? European Child \& Adolescent Psychiatry, 6, 55-72.

Jordan, R. \& Powell, S. (1993). Reflections on the option method as a treatment for autism. Journal of Autism and Developmental Disorders, 23, 682-685.

Kaufman, B. (1976). Son-Rise. New York: Harper-Collins.

Kaufman, B. (1994). Son-Rise: the miracle continues. Tiburon: H. J. Kramer.

Odom, S., Boyd, B., Hall, L. \& Hume, K. (2010). Evaluation of comprehensive treatment models for individuals with autism spectrum disorders. Journal of Autism and Developmental Disorders, 40 (4), 425-436.

Pereira, A., Riesgo, R. S. \& Wagner, M. B. (2008). Autismo infantil: tradução e validação da Childhood Autism Rating Scale para uso no Brasil. Jornal de Pediatria, 84, 487-494. 
Pinto, D., Pagnamenta, A., Klei, L., Anney, R., Merico, D., Regan, R., . . . Carson, A. (2010). Functional impact of global rare copy number variation in autism spectrum disorders. Nature, 466, 368-372.

Prizant, B., Wetherby, M. \& Rydell, P. (2000). Communication intervention issues for children with autism spectrum disorders. In M. Wetherby \& B. Prizant (Eds.). Autism spectrum disorders. A transactional developmental perspective. (pp.193-224). Baltimore: Paul H. Brookes.

Remington, B., Hastings, R. P., Kovshoff, H., Espinosa, F., Jahr, E., Brown, T., . . MacLean, W. (2007). Early intensive behavioral intervention: outcomes for children with autism and their parents after two years. American Journal on Mental Retardation, 112 (6), 418-438.

Rosenwasser, B. \& Axelrod, S. (2002). More contribuitionof applied behavior analysis to the education of people with autism. Behavior Modification, 26 (1), 3-8.

Sandberg, E. \& Spritz, B. (2012). A brief guide to autism treatments. London: Jessica Kingsley.

Schmidt, C. (2012). Temple Grandin e o autismo: uma análise do filme. Revista Brasileira de Educação Especial, 18 (2), pp.179-194.

Schoen, A. (2003). What potencial does the applyed behavior analysis approach have for the treatment of children and youth with autism? Journal of Instructional Psychology, 30 (2), 125-130.

Schopler, E., Reichler, R. \& Renner, B. (1988). The childhood autism rating scale (CARS). Los Angeles: Western Psychological Services.

Schreibman, L. (2007). Theoretical perspectives on behavioral intervention for individuals with autism. In D. L. Cohen \& F. R. Volkmar (Eds.). Handook of Autism and Pervasive Developmental Disorders. (pp. 920-933). New York: Wiley.

Siegel, B. (1996). The world of the autistic child: understanding and treating autistic spectrum disorders. New York: Oxford University Press.

Simpson, R. (2005). Evidenced-based practices and students with autism spectrum disorders. Focus on Autism and Other Developmental Disabilities, 20 (3), 140-149. 
Strain, P. \& Bovey, E. (2011). Randomized, controlled trial of the LEAP model of early intervention for young children with autism. Topics in Early Childhood Special Education, 31 (3), 133-154.

Suplino, I. (2010). Comunicação e inclusão social: análise das contribuições do cinema para o processo de inclusão social. Contemporânea, 8(3), 58-73.

Tolenzani, M. (2011).Inspirados peloautismo. Recuperadoa partir dehttp://www. inspiradospeloautismo.com.br/?gclid=COvciezMvbMCFQSqnQodOicAMA.

Tutt, R., Powell, S. \& Thornton, M. (2006). Educational approaches in autism: what we know about what we do. Educational Psychology in Practice, 22 (1), 69-81.

Welterling, A., Turner-Brown, L., Harris, S., Mesibov, G. \& Delmolino, L. (2011). The home TEACCHing program for toddlers with autism. Journal of Autism and Developmental Disorders, 42 (9), 1827-1835.

Williams, K. (2006). The Son-Rise Program intervention for autism: prerequisites for evaluation. Autism, 10 (1), 86-102.

Williams, K. \& Wishart, J. (2003). The Son-Rise Program intervention for autism: an investigation into family experiences. Journal of Intellectual Disability Research, 47 (4), 291-299. 